\title{
Von der lex divina zur lex positiva im Drama und in der Traktatliteratur des Siglo de Oro
}

\author{
Strosetzki, Christoph
}

First published in:

Zwischen dem Heiligen und dem Profanen, S. 41 - 56, Wilhelm Fink Verlag, München 2008 ISBN 978-3-7705-4616-9

Münstersches Informations- und Archivsystem multimedialer Inhalte (MIAMI) URN: urn:nbn:de:hbz:6-62439445386 


\section{Christoph Strosetzki}

\section{Von der lex divina zur lex positiva im Drama und in der Traktatliteratur des Siglo de Oro}

Die Verankerung der staatlichen Gesetzgebung in nicht verhandelbaren Prinzipien kultureller, ethischer oder religiöser Art wird und wurde ebenso häufig postuliert wie negiert. Während den einen die Identifikation mit der Verfassung ausreicht, fordern die anderen eine Leitkultur, auf deren Grundlage das positive Recht erst zur Geltung kommen könne'. Man hat sich neuerdings daran gewöhnt, den religiös neutralen Rechtsstaat parlamentarischer Prägung dem islamischen Gottesstaat gegenüberzustellen. Ersterer berücksichtige in seiner Gesetzgebung die freie und vernünftige Autonomie mündiger Bürger, letzterer sei auf die Scharia gegründet. Allerdings treten die abendländischen Demokratien erst am Ende eines langen Prozesses der Abstreifung gottesstaatlicher Modelle auf. Dabei hat der Prozess der Aufklärung sicherlich zu einer Säkularisierung geführt, die Frage aber nach der Notwendigkeit der Korrektur positiven Rechts durch übergreifende Prinzipien nicht endgültig beantwortet.

Islamische Rechtswissenschaft in islamischen Glaubensstaaten ist SchariaExegese, wird vom «Obersten Rat für Islamische Angelegenheiten» in Kairo seit Jahrhunderten betrieben und liegt in den 27 Bänden der Enzyklopädie des islamischen Rechts vor. Während es im Koran heißt: "Wer gegen Gott und seinen Gesandten ungehorsam ist, der befindet sich in einem offenkundigen Irrtum», steht im Alten Testament Ähnliches ${ }^{2}$. Die «zehn Gebote» habe Jahwe eigenhändig auf die beiden Gesetzestafeln geschrieben ${ }^{3}$ und Moses übergeben, der sie aus Zorn über sein Volk zerschlug, bevor sie dann in einer zweiten Anfertigung im Tempel von Jerusalem aufbewahrt wurden. Auch das Neue Testament schreibt: «Man muss Gott mehr gehorchen als den Menschen ${ }^{4}$. Wenngleich sich die mittelalterlichen Theoretiker auf die Bibel berufen, finden sie bei der Rückführung menschlicher Gesetze auf göttliche gleichzeitig Unterstützung durch die griechische Philosophie und die römische Stoa.

1 Norbert Lammert (Hrsg.), Verfassung. Patriotismus. Leitkultur. Was unsere Gesellschaft zusammenhält, Hamburg: Hoffmann und Campe, 2006.

2 «Wenn du nun der Stimme des Herrn, deines Gottes, gehorchen wirst, dass du hältst und tust alle seine Gebote [...]», in: Deuteronomium 28, 1 .

3 Vgl. Deuteronomium 4, 13.

4 Koran, 33. Sure, V. 36; Apostelgeschichte 5; vgl. Friedrich Wilhelm Graf, Moses Vermächtnis. Über göttliche und menschliche Gesetze, München: Beck, ${ }^{2} 2006$, S. 16; zur religiösen Problementwicklung im Judentum und im Islam vgl. Rémi Brague, La loi de Dieu. Histoire philosophique d'une alliance, Paris: Gallimard, 2005 (L'esprit de la cité), bes. S. 195-251. 
Insofern sich die Selbstdarstellung des Papstes als vicarius Christi seit dem 12. Jahrhundert und die Vorstellung von ihm als höchstem Priester, als pontifex maximus, in der Renaissance durchsetzte, kam ihm die Zuständigkeit für die gesamte Christenheit zu, und Rechtsmittel gegen seine Entscheidungen waren ausgeschlossen. Mit der Schwäche der weltlichen Herrschaft im 9. und 10. Jahrhundert hatte der Papst zunehmend definiert, nach welchen christlichen Grundsätzen in der res publica christiana zu leben sei. Der hierarchische Instanzenweg von einem Richter zum nächsthöheren bei Berufungen führte zum Papst, dem obersten Richter. Dieses Prozessrecht diente nicht zuletzt der Verwirklichung der päpstlichen Weltherrschaft. «Der Papst sah sich als Inhaber der kaiserlichen Rechte, als wirklicher Kaiser, und damit berechtigt, alle Würdenträger bis hin zum Kaiser nach seinem Belieben abzusetzen ${ }^{5}$. Bis 1300 gilt der Papst als verus imperator und das Kaisertum als ein beneficium, das der Papst als Lehnsherr seinem Lehnsnehmer übertragen oder auch verweigern konnte. 1302 wird mit der Bulle Unam Sanctam die Lehre vertreten, es gebe nur einen Leib der einen Kirche mit einem Haupt, nicht zwei Köpfe wie bei einem Monstrum, so dass sich beide Schwerter, das geistliche und das weltliche, in der Gewalt des Nachfolgers Petri befinden. Die Situation änderte sich vorübergehend im 15. Jahrhundert, als mehrere Päpste miteinander konkurrierten und das Machtvakuum durch Konzilien kompensiert wurde. Beim 5. Laterankonzil in den Jahren 1512 bis 1517 wurde dann entschieden, dass jeder Konzilsbeschluss nur mit Zustimmung des Papstes wirksam wird, wodurch die päpstliche Monarchie erneut den Sieg davontrug ${ }^{6}$.

Welche Verbindungen gibt es zwischen göttlicher und weltlicher Ebene? Die christliche Schöpfungslehre nimmt für Schöpfer und Geschöpf eine gemeinsame Ordnung an und legt so den Ableitungszusammenhang zwischen ius divinum und ius naturale fest. Auf drei Ebenen gilt die Ordnung der lex dei: in der Natur, in den Gesetzen des Staates und in den moralischen Geboten für den einzelnen. Handelt der einzelne also moralisch gut, dann orientiert er sich mit seiner individuellen Vernünftigkeit an der Vernünftigkeit der Schöpfung. Aus dem ius divinum läßt sich ein ius naturale ableiten, das wiederum maßgeblich ist für die einzelnen Gesetze des positiven Rechts. Letzteres steht als vergängliche lex temporalis der als ewig gedachten lex dei gegenüber. Die Ursprünge für die christliche Gesetzesordnung liegen in der Antike. In diesem Zusammenhang kommt Platon und der Stoa, vor allem aber der Aristotelesrezeption des Thomas von Aquin und der Thomasrezeption der politischen Theoretiker im Siglo de Oro, Bedeutung zu. Platon lässt Hippias die Opposition von Natur und Setzung (thesis: woraus sich das Wort «Gesetz» als «Gesetztes» erschließt) folgendermaßen veranschaulichen:

5 Mathias Schmoeckel, Auf der Suche nach der verlorenen Ordnung. 2000 Jahre Recht in Europa; ein Überblick, Köln u. a.: Böhlau, 2005, S. 136; vgl. im folgenden ebd., S. $182 \mathrm{f}$.

6 Vgl. ebd., S. 226. 
Ich denke, sagte er, ihr versammelten Männer, dass wir Verwandte und Befreundete und Mitbürger von Natur sind, und nicht durch das Gesetz. Denn das Ähnliche ist dem Ähnlichen von Natur verwandt, das Gesetz aber, welches ein Tyrann der Menschen ist, erzwingt vieles gegen die Natur ${ }^{7}$.

Die ungeschriebenen Gesetze der Natur erscheinen Hippias als höhere Richtschnur einer Ordnung, deren Gültigkeit unabhängig von bloßer Anerkennung sei, da sie von Natur aus in menschlichen Handlungen wirkten. Daher ist der sich damit beschäftigende Philosoph dem Gesetzgeber gegenüber nicht gleichberechtigt, sondern kritisch und überlegen.

Aristoteles verbindet Gesetz mit Moral und handelt Gerechtigkeit, Recht und Billigkeit als Tugenden im fünften Buch der Nikomachischen Ethik ab. Er unterscheidet dianoetische Verstandestugenden und ethische Charaktertugenden. Grundgebot für alle Tugenden ist die mesotes, die rechte Mitte zwischen dem Zuviel und dem Zuwenig, zum Beispiel steht Tapferkeit zwischen Draufgängertum und Feigheit. Gerechtigkeit wird als ethische Charaktertugend, als Mitte zwischen Unrecht-Tun und Unrecht-Erleiden beschrieben. Ungerechtigkeit ist also die Verletzung einer Proportion, so dass zum Beispiel bei der eigenen Person das Zuviel an Vorteilhaftem und Zuwenig an Nachteiligem Ungerechtigkeit ist ${ }^{8}$. Indem das Gesetz Handlungen verbietet, fördert es im Allgemeinen die Tugend, so wenn es zum Beispiel in der Schlacht die Flucht verbietet, die Tapferkeit, oder wenn es den Ehebruch verbietet, die Besonnenheit. Der aristotelische Rechtsbegriff ist also nicht vom ethischen Kontext abgelöst: Es «darf nicht übersehen werden, was die entarteten Verfassungen tatsächlich übersehen: die Mitte» ${ }^{9}$.

7 Zitiert nach Stefan Lippert, Recht und Gerechtigkeit bei Thomas von Aquin. Eine rationale Rekonstruktion im Kontext der "Summa theologiae», Marburg: Elwert, 2000 (Marburger theologischen Studien, 65), S. 30; Platon, Protagoras, 337 c-d. Dagegen sieht der durch seinen homo-mensura-Satz bekannte Sophist Protagoras bei den Gesetzen das Verhältnis von physis und nomos als Gegensatz und gewährt der menschlichen Gesetzgebung weitgehende Unabhängigkeit.

8 «Beim ungerechten Hergang liegt das Zuwenig im Unrechtleiden, das Zuviel im Unrechttun»; Aristoteles, Nikomachische Ethik, hrsg. v. Günther Bien, Hamburg: Meiner, 1995, S. 115 (1133 b). Vgl. zur Verbindung von Ethos und Gesetzgebung Joachim Ritter, (Naturrecht) bei Aristoteles. Zum Problem einer Erneuerung des Naturrechts, Stuttgart: Kohlhammer, 1961 (Res publica, 6), S. 23.

9 Aristoteles, Politik, hrsg. v. Günther Bien, Hamburg: Meiner, 1995, S. 193 (1309 b); Beim mittelalterlichen Aristotelesdeuter Thomas von Aquin steht die Thematik des Rechts im Zusammenhang mit der Gerechtigkeit, die als Kardinaltugend nach der Klugheit und vor der Tapferkeit und Besonnenheit thematisiert wird. Zur Veranschaulichung der lex aeterna führt Thomas einen Künstler an, der mit seiner ratio gestaltet, wie der Weltregent mit seiner ratio die Ordnung seiner Regierungsgewalt vorgibt. Wenn nun Gesetze Maß und Regel von Handlungen sind, dann erscheint das ewige Gesetz als «Plan der göttlichen Weisheit, insofern [es] alle Handlungen und Bewegungen lenkt». Erkennbar sei die der göttlichen Sphäre zugehörige lex aeterna für endliche Wesen nicht direkt, sondern so wie die Ideen im platonischen Höhlengleichnis nur am Abglanz. Vgl. Thomas von Aquin, Summa Theologica I-II, Das Gesetz I-II, q. 90-105, hrsg. v. Katholischen Akademikerverband, Heidelberg/ Graz u. a.: Kerle/Styria, 1977, Bd. 13, q. 93, 1, S. 48. 
Die Verbindung von Sein und Sollen ergibt sich aus dem Naturbegriff, der bei Aristoteles teleologisch ist. Dabei ist an die Zielursachenlehre zu denken, die das Wesen einer Sache in der immanenten Form sieht, die sie von der Möglichkeit zur Wirklichkeit überführt. Natur ist also zugleich Ziel und Zweck. Die Zielursache strebt den vollkommenen Naturzustand an: «Denn die Beschaffenheit, die ein jedes Ding beim Abschluss seiner Entstehung hat, nennen wir die Natur des betreffenden Dinges, sei es nun ein Mensch oder ein Pferd oder ein Haus oder was sonst immer» ${ }^{10}$. Wenn dies mit der Definition des Menschen als zoon politikon und dem Erstreben der angelegten Möglichkeit des obersten Gutes verbunden wird, dann werden gute Verfassungen zur Bedingung der Möglichkeit menschlicher Entfaltung, da sie am Gemeinwohl ausgerichtet sind, im Gegensatz zu schlechten Verfassungen, in denen Eigennutz die Politik dominiert.

Als eine Anwendung der Moralphilosophie, um das Verhalten der Bürger zu steuern, charakterisiert der spanische Rechtstheoretiker Francisco Suárez 1612 in De legibus die Jurisprudenz" . Er beruft sich auf Platons Dialoge Timaios und Phaidros, wenn er vier Kategorien von Gesetzen unterscheidet: die göttlichen, himmlischen, natürlichen und menschlichen. Da unter den himmlischen Gesetzen die der schicksalsbestimmenden Himmelskörper zu verstehen sind, lehnt Suárez sie ab. Das göttliche Gesetz, das er auch als lex aeterna bezeichnet, charakterisiert er als «ratio gubernatrix universi in Dei mente existens» ${ }^{12}$. Die natürlichen Gesetze sieht Suárez einerseits als Emanationen der göttlichen Gesetze und andererseits als Resultate unmittelbarer menschlicher Einsichten in Gut und Böse ${ }^{13}$. Die vierte Kategorie bilden schließlich die menschlichen Gesetze, das positive Recht. Auch wenn diese direkt vom Menschen verkündet werden, nennt Suárez Plutarch, der auf die Gottähnlichkeit des Fürsten hinweist, und Augustinus, nach dem der weise menschliche Gesetzgeber immer auch die lex aeterna in seine Überlegungen einbeziehe ${ }^{14}$.

Was passiert aber, wenn das positive Recht, wenn also die menschlichen Gesetze, nicht den natürlichen oder göttlichen entsprechen? Aristoteles schreibt dem Naturrecht einen höheren Rang als dem Recht aufgrund von Satzung zu. Zwar können auch in der Natur Veränderungen auftreten, wenn etwa die linke Hand so trainiert wird, dass sie die Aufgaben der rechten über-

10 Aristoteles, Politik (Anm. 9), S. 4 (1252 b).

11 Francisco Suárez, De Legibus I, Madrid: CSIC, 1971 (Corpus Hispanorum de pace, 11), S. 5; vgl. zur Finalität des Menschen als metaphysischer Grundlage des sittlich Guten Elisabeth Gemmeke, Die Metaphysik des sittlich Guten bei Franz Suarez, Freiburg im Breisgau u. a.: Herder, 1961 (Freiburger theologische Studien, 84), S. 51-62.

12 Suárez, De Legibus I (Anm. I1), S. 40; vgl. auch F. S., De Legibus III, Madrid: CSIC, 1974, S. 4-57.

13 Vgl. Gemmeke, Metaphysik des sittlich Guten (Anm. 11), S. 254-264.

14 Suárez, De Legibus I (Anm. 11), S. 54; vgl. P. Alois Schubert, Augustins Lex-AeternaLehre. Nach Inhalt und Quellen, Münster: Aschendorff, 1924 (Beiträge zur Geschichte der Philosophie des Mittelalters, 24/2). 
nehmen kann. Doch was verändert werden kann, darf es nicht unbedingt. Wenn es naturrechtlich geboten ist, die Toten zu bestatten, kann auch entgegengesetztes positives Recht daran nichts ändern, wie Aristoteles in seiner Rhetorik am Beispiel des Dramas Antigone von Sophokles ausführt ${ }^{15}$. Nachdem König Kreon verfügt hat, dass Antigones abtrünniger Bruder Polyneikes nicht nach Recht und Brauch begraben werden darf, sondern den Vögeln zum Fraß vorgeworfen werden soll, gibt er als allgemeines Gesetz bekannt, dass Zuwiderhandelnde öffentlich gesteinigt werden sollen. Antigone aber sieht sich mehr an das natürliche bzw. göttliche als an das menschliche Gesetz gebunden und widersetzt sich daher Kreons Gesetz: «Auch glaubte ich, so viel vermöchte kein Befehl von dir, um ungeschriebne, ewige, göttliche Gesetze zu überrennen als ein Sterblicher. Denn nicht von heut und gestern, sondern immerdar bestehn sie: niemand weiß, woher sie kommen sind» ${ }^{16}$. Auch die Stoiker thematisieren die mögliche Inkongruenz von überpositivem und positivem Gesetz. Sie führen eine göttliche Weltvernunft ein, die sie nomos nennen und deren Gesetze von der menschlichen Gesetzgebung abzugrenzen sind. Sie unterscheiden daher zwischen dem Weltgesetz (lex aeterna), dem Naturgesetz (lex naturalis) und dem vom Menschen gesetzten Gesetz (lex humana). Da es das Weltgesetz erforderlich macht, sich in das Weltgeschehen einzufügen, wird nach Chrysipp der, der sich dagegen auflehnt, ebenso erfolglos bleiben wie der Hund, der sich auf die Hinterbeine setzt und doch mitgeschleift wird ${ }^{17}$. Ebenso wie das Weltgesetz des Kosmos ist nach dem Stoiker Cicero die lex naturalis, deren anthropologische Konstanten zum Beispiel Pflichterfüllung und Verbot von Unredlichkeit sind, der menschlichen Verfügbarkeit entzogen:

Wir können aber auch nicht durch den Senat oder das Volk von diesem Gesetz gelöst werden, es braucht als Erklärer und Deuter nicht Sextus Aelius geholt werden, noch wird in Rom ein anderes Gesetz sein, ein anderes in Athen, ein anderes jetzt, ein anderes später, sondern alle Völker und zu aller Zeit wird ein einziges, ewiges und unveränderliches Gesetz beherrschen, und einer wird der gemeinsame Meister gleichsam und Herrscher aller sein: Gott ${ }^{18}$.

Gott, Natur und Vernunft befinden sich also auf einer höheren, nichtempirischen Warte, die die positiven Gesetze transzendiert und kritisch betrachtet. Nur eine lex naturalis bietet das Kriterium, gute von schlechten positiven Gesetzen zu unterscheiden. Daher ist es töricht «zu glauben, alles sei gerecht, was in Bestimmungen und Gesetzen der Völker fest gelegt ist. Etwa

15 Aristoteles, Rhetorik, 1373 b; vgl. Lippert, Recht und Gerechtigkeit (Anm. 7), S. 54.

16 Sophokles, Antigone, gr./dt., hrsg. v. Bernhard Zimmermann, Düsseldorf/Zürich: Artemis \& Winkler, 1999 (Tusculum Studienausgabe), S. 37, V. $453 \mathrm{ff}$.

17 Vgl. Hans Welzel, Naturrecht und materiale Gerechtigkeit, Göttingen: Vandenhoeck \& Ruprecht, ${ }^{4} 1962$ (Jurisprudenz in Einzeldarstellungen, 4), S. 40

18 Cicero, De re publica / Vom Gemeinwesen, lat./dt., hrsg. v. Karl Büchner, Stuttgart: Reclam, 1995, S. 281 (III, 22). 
auch, wenn es irgendwelche Gesetze von Tyrannen sind» ${ }^{19}$. Ungerechte menschliche Verordnungen sind für Cicero ebensowenig Gesetze wie Übereinkünfte von Räubern.

Würde sich aber das Recht nur auf die Weisungen der Völker, die Anordnungen der Verantwortlichen und die Entscheidungen der Richter stützen, dann wäre es Recht zu rauben, die Ehe zu brechen und Testamente zu fälschen, wenn dies nur durch Abstimmungen und Beschlüsse einer Mehrheit gebilligt würde ${ }^{20}$.

Die Trennung der lex humana vom natürlichen und göttlichen Gesetz und damit die Autonomisierung des empirischen und positiven Rechts wird auch in der Rechtstheorie des Aristotelikers Thomas von Aquin problematisiert ${ }^{21}$. Empirisch festhalten lässt sich die Unfähigkeit des Menschen, als einzelner gemäß seinen Interessen zu leben: "Wären nämlich viele Menschen beisammen und jeder nur auf das bedacht, was ihm selbst angemessen erscheint, so würde die Gesellschaft nach entgegengesetzten Richtungen auseinandergeraten ${ }^{22}$. Metaphysisch wird als Gegenmittel die Notwendigkeit von Recht und Staat postuliert. Ergab sich diese bei Platon aus der Idee der Gerechtigkeit, wird sie bei Thomas aus dem göttlichen Willen abgeleitet. Und nicht weil viele etwas wollen und daraus Gesetze machen, ist der faktische Wille der Menge zugleich ein Sollen. Wäre das positive Recht schon deshalb gerecht, weil es aus dem Willen des Menschen hervorgeht, dann könnte der menschliche Wille nicht ungerecht $\operatorname{sein}^{23}$. Lex humana bezeichnet nach Thomas das positive Recht, das aus den beiden übergeordneten Ebenen, lex aeterna und lex naturalis, abzuleiten ist. Wo die Ableitung von der oberen auf die untere Ebene nicht möglich ist, sieht Thomas Freiräume für die eigenständige Entscheidung entstehen.

Der Humanist Luis de León unterscheidet in seiner rechtstheoretischen Schrift De legibus 1571 im Anschluss an die Stoa und an Thomas' ewiges, natürliches und menschliches Gesetz. Die zahlreichen Vorschriften des natürlichen Gesetzes sind auf die Weisung zu reduzieren, das Gute zu tun und das Böse $\mathrm{zu}$ meiden, sowie naturgemäß zu leben ${ }^{24}$. Auch wenn menschliche

19 Cicero, De legibus / Über die Gesetze, lat./dt., hrsg. v. Rainer Nickel, Düsseldorf/Zürich: Artemis \& Winkler, ${ }^{2} 2002$, S. 46 f. (De legibus I, XV, 42).

20 Ebd., S. $48 \mathrm{f}$. (De legibus I, XV, 43); zu Cicero als Vorläufer für Thomas vgl. Wolfgang Kluxen, ¿Lex naturalis` bei Thomas von Aquin, Wiesbaden: Westdeutscher Verlag, 2001, S. 16 (Nordrhein-Westfälische Akademie der Wissenschaften, G 378).

21 Vgl. Thomas von Aquin, Summa Theologica I-II. Das Gesetz I-II (Anm. 9), q. 90-105; Summa Theologica I. Erhaltung und Regierung der Welt, q. 103-1 19, hrsg. v. Katholischen Akademikerverband, Heidelberg/Graz u.a.: Kerle/Styria, 1951, Bd. 8; Summa Theologica II-II. Recht und Gerechtigkeit, q. 57-79, hrsg. v. Katholischen Akademikerverband, Heidelberg/Graz u. a.: Kerle/Styria, 1953, Bd. 18.

22 Thomas von Aquin, Über die Herrschaft der Fürsten, übers. v. Friedrich Schreyvogl, Stuttart: Reclam, 1971, S. 7.

23 Summa Theologica II-II, q. 57, 2; S. 7 f. (Anm. 21).

24 Fray Luis de León, De legibus (1571), hrsg. v. Luciano Pereña, Madrid: CSIC, 1963, S. 68 (Corpus Hispanorum de pace, 1). 
Gesetze nach Luis de León im allgemeinen vom natürlichen oder ewigen Gesetz abgeleitet sind, kann es doch Ausnahmen geben, wie zum Beispiel bei den Universitätsstatuten von Salamanca, beim Getreidepreis oder der Residenzpflicht von Klerikern ${ }^{25}$. Vor diesem Hintergrund kann ein Gesetz auch fehlschlagen und damit die Frage aufwerfen, ob es den Untertanen erlaubt ist, dem Gesetz zuwiderzuhandeln. Luis de León hält den zivilen Ungehorsam für gerechtfertigt, wenn mit Sicherheit feststeht, dass bei Gesetzesbefolgung das bonum commune verletzt wird ${ }^{26}$. Auf das Prinzip des bonum commune als Kriterium zur Bewertung von positivem Recht soll später noch eingegangen werden.

Es gibt also einzelne Fälle, in denen ziviler Ungehorsam gegenüber falschen Gesetzen erlaubt ist. Denkbar ist auch, dass die gesamte positive Gesetzessystematik fehlgeleitet ist, wenn sie sich etwa im Gegensatz zum menschlichen bonum commune oder zum göttlichen Gesetz befindet. So ist zum Beispiel die Finalität dann nicht gegeben, wenn sich ein Gesetz nicht am Gemeinwohl, sondern am Eigennutz des Legislators ausrichtet. Die positiven Gesetze stehen dann deutlich im Gegensatz zum göttlichen Gut, wenn sie zur Gottlosigkeit verleiten. Hier begründet Thomas die Widerstandspflicht kategorisch mit dem Hinweis auf Paulus' von uns bereits anfangs erwähnten Satz, man solle Gott mehr gehorchen als den Menschen ${ }^{27}$. Ist das Ende der Tyrannei absehbar und die Intensität der Unterdrückung erträglich, dann kann jedoch von einem Umsturz im Interesse der Beibehaltung der Rechtssicherheit abgesehen werden. Sind Temporalitäts- und Intensitätsvorbehalte nicht erfüllt, dann gilt nach Thomas:

Wenn es zum Rechte eines Volkes gehört, sich selbst einen König zu bestimmen, so kann mit vollem Rechte der eingesetzte König von ebendemselben Volke von seinem Platze entfernt oder seine Macht eingeschränkt werden, wenn er die königliche Gewalt in tyrannischer Weise missbraucht. Und man darf nicht glauben, dass ein solches Volk gegen die Treue handelt, indem es den Tyrannen absetzt ${ }^{28}$.

Es stellt sich die Frage, wann das Absetzen cines Tyrannen und der Volksaufstand erlaubt sind. Sie wird konkret in der Quaestio de seditione des Humanisten und Thomisten Francisco de Vitoria (1492-1546) diskutiert. Der Volksaufstand sei insofern mit Krieg vergleichbar, als es sich um eine Konfrontation mit Angriff und Verteidigung handle. Der Unterschied bestehe jedoch darin, dass beim Krieg eine gegenseitige Konfrontation vorliege, während es beim Volksaufstand ausreiche, wenn eine Seite die Waffen erhebe. Ein weiterer Unterschied liege darin, dass der Krieg gegen Fremde und Feinde geführt werde, der Volksaufstand zwischen Parteiungen einer einzigen Gemeinschaft stattfinde. Gibt es zum Beispiel in einer Stadt einen Tyrannen und

25 Ebd., S. 87, 112-118.

26 Ebd., S. 125.

27 Lippert, Recht und Gerechtigkeit (Anm. 7), S. 154.

28 Thomas von Aquin, Über die Herrschaft der Fürsten (Anm. 22), S. 24. 
es erhebt sich eine Partei gegen ihn und eine andere für ihn, dann kommt es zum Aufstand, wie etwa in Florenz bei den Anhängern und Gegnern der Medici. Wenn Vitoria dem Volk das Recht zubilligt, die Tyrannei zu beendigen, greift er auf Thomas von Aquin zurück. So habe sich auch der Apostel Petrus gegen die Saduzäer und gegen die Pharisäer gestellt, «Quia concordia eorum erat mala. Ita etiam concordia populi in tyrannide potest esse mala et ideo aliquis potest tollere talem concordiam ${ }^{29}$. Nicht jede Einheit und jeder Friede sind es also wert, geschützt zu werden. Wie auch nicht jede Menschenmenge, etwa die von Dieben, als Volk zu bezeichnen ist. Unter Rückgriff auf Augustinus definiert Vitoria das Volk als «coetum iuris consensu et utilitatis communione sociatum» ${ }^{30}$. Mit Thomas rät Vitoria zur Wahrung der Verhältnismäßigkeit und dazu, den Tyrannen zu dulden, wenn andernfalls mehr als die Hälfte der Bürger einer Stadt dem Aufstand zum Opfer fallen würde.

Darf ein beliebiger Privatmann den Tyrannen töten? ${ }^{31}$ Dagegen spricht nach Vitoria, dass ein Privatmann nicht durch öffentliche Autorität legitimiert ist. Dafür spricht, dass man sich gegenüber einem Angreifer verteidigen kann und dass im Rahmen einer legitimen Verteidigung Gewalt mit Gewalt beantwortet werden darf. Umso mehr ist es erlaubt, denjenigen zu töten, der die Republik angreift. Zu bedenken gibt Vitoria, dass zwei Arten von Tyrannen zu unterscheiden sind: Der eine ergreift die Königskrone, ohne dazu berechtigt zu sein, der andere ist zwar berechtigt, wird aber im Verlauf seiner Regierungszeit zum Tyrannen, der alles dem eigenen Vorteil und nicht dem Gemeinwohl zuordnet und damit den Staat ruiniert. Gegen den zweiten Typ darf sich der Staat wehren, nicht aber eine Einzelperson. Da gegen den ersten Typ der Staat immer schon Krieg führe, dürfe in diesem Fall auch eine Privatperson töten, da sie dann im Interesse der Verteidigung des Staates handle und durch öffentliches Interesse legitimiert wäre.

Luis de Montesinos, der zwischen 1593 und 1620 an der Universität von Alcalá lehrte, führt weitere systematische Gründe zur Rechtfertigung zivilen Ungehorsams an ${ }^{32}$. Zwar sci Gehorsam ein Gebol des Naturrechts, jedoch nur unter der Voraussetzung, dass sich der Herrscher am Gemeinwohl orientiert. Nur wenn er dem Ganzen diene und die Freiheit der Untergebenen garantiere, sei politischer Gehorsam gerechtfertigt. In jedem anderen Fall werde ziviler Ungehorsam zur Pflicht. Auch sei Gehorsam kein absoluter Wert, sondern er

29 Francisco de Vitoria, "Quaestio de seditione», in: F. de V., Relectio de Iure Belli o Paz Dinámic.: Escuela Española de la Paz primera generación 1526-1560, hrsg. v. Luciano Pereña, Madrid: CSIC, 1981, S. 274 (Corpus Hispanorum de pace, 6), («porque el estar de acuerdo con ellos era malo. Asi también el estar de acuerdo el pueblo con la tiranía puede ser malo y por eso puede uno acabar con esa unión»).

30 Ebd., S. 270 («sino una asociación estructurada sobre una aceptación común del derecho y sobre la comunidad de intereses»).

31 Francisco de Vitoria, "Dubium de tyranno", in: Vitoria, Relectio (Anm. 29), S. 279-285.

32 Vgl. Luciano Pereña, «Perspectiva histórica», in: Francisco Suárez, De iuramento fidelitatis, hrsg. v. Luciano Pereña, Madrid: CSIC, 1979, S. 128-130 (Corpus Hispanorum de pace, 18). 
stehe in Relation zur Legitimität der staatlichen Autorität, zum rechten Machtgebrauch, zum Grad der Orientierung am Gemeinwohl sowie zu Recht und Gerechtigkeit gegenüber allen Untertanen. Je größer hierbei Mängel hervortreten, desto berechtigter werde ziviler Ungehorsam. Da die Macht der Könige vom Volk stamme und dieses der Übertragung an den Souverän zuzustimmen habe, sei man einem Despoten, der durch Gewalt an die Macht gekommen ist, nicht zu Gehorsam verpflichtet. Auch wo das Gemeinwohl in offensichtlicher Weise verletzt werde, die Untertanen unterdrückt und versklavt werden und die Gesetze nicht mehr dem Gemeinwohl, sondern dem Herrscher dienen, ist nach Luis de Montesinos ziviler Ungehorsam geboten. Oberstes Prinzip ist also das Gemeinwohl, an dem die Herrscher ebenso orientiert sein sollen wie die Beherrschten. Ein Verstoß dagegen durch den Herrscher erscheint als Vertragsbruch, der auch den Beherrschten aus seinen Bindungen entlässt ${ }^{33}$.

Zur konkreten Veranschaulichung sei auf Calderón de la Barca eingegangen. In seinem Drama Amar después de la muerte ${ }^{34}$ werden der Aufstand und die Sezession der Morisken dadurch veranlasst, dass König Philipp II. verordnet, kein Moriske dürfe Nationalfeste abhalten, seidene Kleider tragen, in öffentlichen Bädern gesehen werden, sich frei mit anderen in seinem Haus versammeln oder sich der arabischen Sprache bedienen ${ }^{35}$. Nun denken die Morisken, die Christen wollten sie versklaven, und ziehen mit Waffen und Lebensmitteln in das Alpujarra-Gebirge, um dort nicht als Sklaven, sondern als freie Herren zu leben. Die Morisken ernennen ihrerseits einen Anführer, und es kommt zu kriegerischen Auseinandersetzungen mit den Spaniern.

33 Ähnlich sieht Diego de Mesa in seinem 1622 beendeten Werk Politica o razón de Estado: «El menosprecio es cuando los que gobiernan, soberbios y desvanecidos, no estiman a los súbditos, tratándolos como a gente vil y con violencia; de lo cual los súbditos desdeñados, como les llega una ocasión que mueva de verás, toman las armas y quitan el dominio a los que lo tienen.) Diego Pérez de Mesa, Política o razón de Estado, hrsg. v. Luciano Pereña, Madrid: CSIC, 1980, S. 201 (Curpus Hispanorum de pace, 20); «La desemejanza asimisma causa alteración y mutación de estados; en la cual principalmente entra la desemejanza y diferencia en la religión, unos católicos y otros herejes, unos luteranos y otros calvinistas, unos cristianos viejos y otros moriscos. La cual división y disimilitud cuánto sea pestilente y dañosa a todo género de estado, lo manifiestan bien las turbaciones intestinas de Francia, las guerras y azotes de Flandes y Alemania y Inglaterra»; ebd., S. 206. Vgl. auch zum «derecho de resistencia" bei einem spanischen Juristen des 16. Jahrhunderts Francisco Carpintero Benítez, Del derecho natural medieval al derecho natural moderno. Fernando Vázquez de Menchaca, Salamanca: Universidad, 1977, S. 172-181 (Acta Salmanticensia, 35).

34 Zum Vergleich mit einschlägigen Chroniken von Diego Hurtado de Mendoza und Pérez de Hita vgl. Jorge Checa, «Calderón de la Barca y las catástrofes de la historia: Amar después de la muerte», in: Nueva revista de filologia hispánica 51 (2003), H. 1, S. 147-192, hier S. 147 f., S. $182 \mathrm{ff}$.

35 «Ninguno de la nación avricana, que hoy es caduca ceniza de aquella invencible llama en que ardió España, pudiese tener fiestas, hacer zambras, vestir sedas, verse en baños, juntarse en ninguna casa, ni hablar en su algarabía, sino en lengua castellana»; Pedro Calderón de la Barca, "Amar después de la muerte», in: Calderón, Obras completas, hrsg. v. Angel Valbuena Briones, Madrid : Aguilar, ${ }^{5} 1966$, Bd. 1, S. 351-386, hier S. 352. 
Schließlich wird dem spanischen Befehlshaber ${ }^{36}$ zur Milde geraten. Verkündet wird ein Generalpardon, das der Moriskenanführer nicht akzeptiert, da er inzwischen zum «rey tirano» (116) geworden ist. Die Parteien der Befürworter und Gegner dieser Entscheidung beginnen einen Bürgerkrieg, in dessen Verlauf sie ihren Anführer ermorden. Während sich also zunächst die Morisken berechtigterweise gegen die Spanier erheben, schwächen sie schließlich durch einen Bürgerkrieg die eigenen Reihen.

Gleich zu Beginn des Dramas wird auf die religiösen Sitten der Mauren hingewiesen: "Celebremos nuestro día, / que es el viernes, a la usanza / de nuestra nación, sin que / pueda esta gente cristiana, / entre quien vivimos hoy / presos en miseria tanta, / calumniar ni reprender / nuestras ceremonias» ${ }^{37}$. Dreimal werden Allah und die gloreiche maurische Vergangenheit evoziert, dreimal schließt sich dabei der Ausruf aller Umstehenden Morisken an: «Su ley viva! ${ }^{38}$. Gemeint ist damit die lex divina des Islam. Sie eröffnet also die erste Szene des ersten Aktes, vor deren Hintergrund dann der Ungehorsam gegenüber der spanischen lex positiva beschlossen werden kann. Dennoch lässt Calderón - wohl auch mit Rücksicht auf die Zensur - den Sprecher der Morisken Malek die Anordnung vorsichtig als «ley justa y prevención santa» bezeichnen, da es natürlich sei, dass die afrikanischen Sitten allmählich in Vergessenheit gerieten, jedoch dürfe man bei einer Veränderung der Sitten nicht "con furia tanta» und "violencia» vorgehen ${ }^{39}$. Angesichts der Härte der Spanier bleibt nur die Sezession in die Alpujarra, die die Morisken noch als ihren eigenen Besitz betrachten: «toda es nuestra: retiremos a ella bastimentos $y$ armas. [...] y haceos señores, de esclavos» ${ }^{40}$. Schließlich ist es Isabel Tuzani, die auf den Generalpardon der Spanier vertrauend den Aufstand beendet. Bedenkt man nun, dass sie ihrerseits als «morisca en la voz y católica en el almas " ${ }^{41}$ lebte, dann ermöglicht sie zugleich einen spielerischen Kontrapunkt zur Ausgangssituation des Dramas und baut eine Brücke zur Einsicht in die Legitimität des Handelns der Morisken. Die bei ihr und den Morisken bestehende Inkongruenz von lex positiva und lex divina löst sie nicht durch Aufstand, sondern durch Aufgabe des Aufstands.

Auch Beispiele für den Tyrannenmord sind bei Calderón nicht selten. Als sich in La gran Cenobia das Kriegsglück zu Aurelians Ungunsten wendet, stürat er wütend die Weissagerin, die ihm einen Sieg prophezeite, in eine Hôhle, woraufhin sie in ihm einen Tyrannen sieht, den sie ihrerseits stürzen will. Als Aurelian gegenüber Decius, der ihm während des Kampfes in einer schwierigen Lage das Leben rettet und dem er einen Anteil am Thron ver-

\footnotetext{
36. Vil. zur Identifizierung der spanischen Fühnung mit der Providenz Checa, «Calderón de la Barcan (Anm. 34). S. 163.

37 Pedro Calderón de la Barca Anwar quespanes de la muserte (Anm. 35), S. 351.

is Elind.

3.4) Ebd. S. 352.

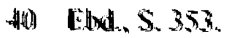

4 Eod $\$$. 35 .
} 
sprochen hatte («te doy palabra de hacerte igual en mi imperio» ${ }^{42}$ ), das Versprechen bricht und auch die lästigen Soldforderungen seiner Soldaten abweist: «¿Qué importa a un rey que haya pobres en su imperio? Sufran y padezcan, pues; que pues el cielo los hizo pobres, él sabe por qué»» ${ }^{43}$, wächst die Zahl seiner Gegner. Auch Livius und seine Freundin Irene wollen ihn ermorden. Decius tut es mit der Begründung: «Muerte mis manos te den por bárbaro, por tirano, por soberbio y por cruel ${ }^{44}$. Alle sind dem Tyrannenmörder dankbar: «Te nombramos César nuestro, por haber librádonos de un tirano» ${ }^{45}$. Hatte sich doch Aurelian weder an der lex divina, noch an der lex naturalis, weder an der Moral, noch am Gemeinwohl orientiert. Auf die Problematik des schon mehrfach erwähnten Gemeinwohls soll nun abschlieBend noch kurz systematisch eingegangen werden.

Das positive Gesetz soll nach Thomas auf das bonum commune hingeordnet sein ${ }^{46}$. Die notwendige Ausrichtung des positiven Gesetzes auf das bonum commune bringt es mit sich, dass positive Gesetze, die dem bonum commune abträglich sind, nicht befolgt werden dürfen, also ein Handeln contra legem geboten ist. Allgemein kann es zur Entfernung des Gesetzes vom bonum commune kommen, wenn es dem menschlichen Legislator nicht möglich war, alle möglichen Einzelfälle zu bedenken, wenn sich die Vernunft weiter vom Unvollkommenen zum Vollkommenen entwickelt hat oder wenn sich die Lebensverhältnisse (conditiones) der Menschen geändert haben. Da die alten Gesetze bereits durch die Gewohnheit (consuetudo) gestützt sind, ist die Bewahrung von Gesetzen zu bevorzugen und Veränderung nur als Reaktion auf deutliche Beeinträchtigung des allgemeinen Wohls zu rechtfertigen ${ }^{47}$. Menschliche Gesetze müssen also wechseln, wenn die Umstände und Verhältnisse, in die sie regulierend eingreifen, sich verändern oder wenn im Zuge der allgemeinen Vervollkommnung «aliquid melius» ${ }^{48}$ in Erscheinung tritt. In diesem Zusammenhang diskutiert Luis de León, ob eine Gewohnheit (consuetudo) Gesetzeskraft erlangen kann, ob eine öffentlich verbreitete Gewohnheit Gesetze interpretiert, ob sie Gesetze abschaffen kann, die etabliert und gebilligt waren, und schließlich, ob eine Gewohnheit von sich aus und ohne schriftliches Zeugnis ein ungeschriebenes Gesetz sein kann. Hier zeigt sich deutlich eine andere Orientierung in der Ableitung der Gesetze: Sie erfolgt nicht deduktiv aus der lex aeterna, sondern induktiv aus der Gewohnheit.

42 Pedro Calderón de la Barca, «La gran Cenobia», in: Obras completas, hrsg. v. Luis Astrana Marín, Madrid: Aguilar, ${ }^{3} 1951$, Bd. 1, S. 155-185, hier S. 171.

43 Ebd., S. 182.

44 Ebd., S. 184.

45 Ebd., S. 184.

46 Thomas von Aquin, Summa Theologica I-II (Anm. 21) q. 95, 4; S. 105 (aber auch schon q. 90,3 ; S. 12).

47 Lippert, Recht und Gerechtigkeit (Anm. 7), S. 145 f.

48 Fray Luis de León, De legibus (Anm. 24), S. 128. 
Da in jedem Fall jedes Gesetz als Ziel das «bonum publicum et commune ${ }^{49}$ hat, leitet Luis de León daraus ab, dass die Vorschriften, die sich am Eigennutz orientieren, keine Gesetze sein können. Denn Könige seien nicht die Eigentümer des Besitzes ihrer Untertanen und können ihn nicht für sich beanspruchen. Da der Herrscher seine Macht vom Volk erhält, hat sie den Zweck, dem Volk zu dienen und zu nutzen. «Nam reges non habent dominium nec imperant servis, sed hominibus liberis» ${ }^{50}$. Dem bonum commune dient auch die Wirkung des Gesetzes, den Menschen vollkommener und moralisch gut zu machen. Dabei stehen dem Gesetz drei Handlungsmöglichkeiten zur Verfügung: «imperare, prohibere, permittere et punire» ${ }^{51}$.

Folgerichtig sieht Francisco Suárez das Gesetz an eine Gemeinschaft und nicht an einen einzelnen gerichtet. Damit es gerecht ist, habe es - hier zitiert Suárez Isidor - «ad commune bonum», d. h. nicht am Einzelinteresse, sondern am gemeinsamen Nutzen für die Bürger, ausgerichtet zu sein ${ }^{52}$. Dabei kann, wie in Suárez' folgendem Aristoteles-Zitat, an der Stelle des Gemeinwohls der Bürger auch der Staat selbst stehen: «Leges ad rem publicam esse accommodandas, non rem publicam ad leges ${ }^{53}$. Suárez beruft sich auf Platon, bei dem Gesetze auf Tugenden und «ad communem pacem et felicitatem» ${ }^{54}$ ausgerichtet seien ${ }^{55}$. Oberste Prinzipien des Gesetzes sollen sein «bonum commune» und "felicitas civitatis»: «Debet ergo esse lex propter commune bonum ${ }^{56}$. Von diesen auf das Gemeinwohl bezogenen Gesetzen sind diejenigen zu unterscheiden, die sich an eine Berufsgruppe oder an Minderjährige wenden. Obwohl sich die Strafgesetze an Straffällige, die Steuergesetze an bestimmte Einkommensgruppen richten, ist auch bei ihnen das «commune bonum» letztes Ziel.

Die Lehre vom Gemeinwohl ist es, die Machiavelli zum Ausgangspunkt seiner Überlegungen macht, wenn er eine unmoralische Handlung des Monarchen dadurch rechtfertigt, dass sie dem Staatswohl und nicht dem Einzelinteresse des Fürsten dient. In seiner Argumentation verzichtet er auf den nicht-empirischen Teil der Rechtslehre. Die zentrale Frage ist bei ihm die nach den Mitteln, mit denen Macht erhalten werden kann. Er beobachtet seine Zeitgenossen und gewinnt ein negatives Bild von ihnen: Sie seien schlecht und

49 Ebd., S. 22; Unabhängig davon haben sich die Gesetze auch am «bonum divinum» auszurichten, da dahin ohnehin das gesamte Universum orientiert ist (vgl. ebd., S. 24).

50 Ebd., S. 31.

51 Ebd., S. 55.

52 Suárez, De Legibus I (Anm. 11), S. 104.

53 Ebd., S. 129.

54 Ebd., S. 130.

55 Vgl. zur Mittelstellung der Tugend bei Aristoteles, Thomas und Suárez Wilhelm Ernst, Die Tugendlehre des Franz Suárez, Leipzig: St.-Benno-Verlag, 1964, S. 204-218 (Erfurter theologische Studien, 15).

56 Suárez, De Legibus I (Anm. 1I), S. 132. 
stets von bösen Neigungen getrieben ${ }^{57}$. Der Mensch benötigt nach Machiavelli den Staat, um sich vor anderen zu schützen. Der Staat setzt also zur Ordnung des Gemeinwesens Zwangsmittel ein, zu denen Gesetze und Gewalt gehören ${ }^{58}$.

Noch einen anderen Punkt gilt es bei Machiavelli festzuhalten: Die Lehre von der Staatsräson ermöglicht es dem stärkeren Individuum oder dem stärkeren Staat, sich ohne Rücksicht auf lex divina oder lex naturalis gegenüber anderen im Interesse des Gemeinwohls durchzusetzen. Das brachte ihm viel Kritik ein, obwohl sein Ansatz weder so neu noch so originell war, wie er schien. Bereits Cicero setzt sich mit dem Skeptiker Karneades auseinander, für den nicht die Natur das Gesetz festlegt, sondern die jeweiligen wechselnden Interessen der Menschen. Für ihn ist natürliche Gerechtigkeit inexistent bzw. Zeichen größter Dummheit, da sie für fremden Vorteil sorge und dem eigenen schade. Als Beispiel werden die Römer angeführt, die, wollten sie gerecht sein, fremdes Gut zurückerstatten und in ihre Hütten zurückkehren müssten. «Kein Staat ist so dumm, dass er nicht lieber ungerecht herrschen als gerecht Sklave sein wollte» ${ }^{59}$. Klug dagegen sei es, zitiert Cicero diese Position weiter,

die Macht zu vergrößern, den Reichtum zu vermehren, die Grenzen vorzuschieben, [...] über möglichst viele zu gebieten, Vergnügungen zu genießen, stark zu sein, zu regieren, zu herrschen; die Gerechtigkeit aber schreibt vor, alle zu schonen, für das Menschengeschlecht zu sorgen, einem jeden das Seine zu geben $[\ldots]^{60}$.

Nach Cicero weist Laktanz darauf hin, dass, wer eine fehlerhafte Sache verkaufen möchte, gerecht ist, wenn er den Fehler angibt, und klug, wenn er ihn verschweigt und Erfolg hat. Noch einen Schritt weiter geht nach Platon der Sophist Kallikles, wenn er das Naturrecht umdeutet, das Gerechte als das Recht des Stärkeren definiert und erklärt, von Natur aus sei das Unrechtleiden,

57 Machiavelli, Il principe / Der Fürst, ital./dt., hrsg. v. Philipp Rippel, Reclam: Stuttgart, 1995, S. 17 (1. Buch, 3. Kap.); später vertrat Hobbes in seinem Leviathan eine vergleichbare Position, als er den Satz «homo homini lupus» prägte.

58 Ebd., S. 77, 129, 135. Wenn allerdings Machiavell im Schlusskapitel von Il Principe Italien von den Barbaren befreien will und ihnen das richtige Recht und gute Gesetze gegenüberstellt, dann befindet er sich insofern auf der Ebene metaphysischer Voraussetzungen, als er der Ordnung gegenüber der Unordnung den Vorzug gibt. Spekulativer noch wird Machiavelli, wenn er darstellt, dass sich in der Frühzeit die Menschen zusammenschlossen, um den Stärksten zu ihrem Anführer zu machen: «Um Übel zu vermeiden, entschloss man sich, Gesetze zu schaffen und Strafen gegen Zuwiderhandelnde einzuführen»; ebd., S. 13. Gleich zwei Mal nennt Machiavelli hier Zwecke, die er seinem Rechtsrealismus als evidente Voraussetzungen stillschweigend zugrundelegt, ohne ihre mögliche Provenienz aus einer lex aeterna oder lex naturalis zu erwähnen. Dabei sieht es so aus, als ob aus dem Sein das Sollen ableitbar ist: Ist der Mensch schlecht, dann soll er durch Gesetze unter Kontrolle gehalten werden. In Wirklichkeit geht der Rechtsrealismus von der nicht-empirischen und einfach gesetzten Voraussetzung aus, dass der Mensch in geordneter Gemeinschaft leben soll.

59 Cicero, De re publica /Vom Gemeinwesen (Anm. 18), S. 277 (III, 18).

60 Ebd., S. 273 (III, 15). 
vom Gesetz aus das Unrechttun übler. Dem Stärkeren gebühre nach der Natur das Herrschaftsrecht über den Schwachen, das ihm der Schwache durch die menschliche Gesetzgebung wegnehme, indem sie ihm die Notwendigkeit der Gleichheit aller suggeriere ${ }^{61}$.

Anschauliches Beispiel für Missachtung des Gesetzes und Arroganz des jeweils Stärkeren bietet Calderóns Stück La cisma de Inglaterra, wo das Recht des Stärkeren als auch ohne Rekurs auf Gemeinwohl und Staatsräson herrscht. Dies zeigt sich beim König Heinrich VIII. ebenso wie bei seinem Kanzler Wolsey und seiner Geliebten Anna Boleyn. In letztere verliebt sich der König, als sie ihm am Hof vorgestellt wird. Sein ehrgeiziger Kardinal Wolsey, dem es nicht gelungen war, Papst zu werden, schwört gegenüber dem neuen Papst Rache. Als die Königin Catalina ihm einmal den Zutritt zum Zimmer des Königs verweigert, schwört er auch ihr Rache. Er verspricht Anna, sie zur Königin zu machen, wenn sie schwöre, ihm gegenüber nie undankbar zu werden, was sie verspricht. Wolsey rät ihr, dem König gegenüber Liebe zu heucheln und ihm zu erklären, sie könne ihn nur lieben, wenn er sie heirate. Dem König erklärt er, seine Heirat mit der Königin Catalina sei ungültig. Heinrich verkündet daraufhin die Ungültigkeit seiner Heirat dem Parlament mit dem abschließenden Hinweis: "Y el vasallo que sintiere mal, advierta temeroso que le quitaré al instante la cabeza de los hombros» ${ }^{62}$. Anna wird Königin und Heinrich bricht mit der katholischen Kirche und ihren Gesetzen. Als Wolsey seine Belohnung von Anna einfordert, lässt sie ihn fallen, worauthin ihn auch der König als Kanzler entläßt. Gewissensbisse hat sie nicht: «¿Tirana me llaman? ¿Ingrata soy?» ${ }^{63}$. Als Anna schließlich mit ihrem Geliebten Carlos zusammentrifft, bemerkt der König, dass er getäuscht worden ist, und verurteilt Anna zum Tode. Als am Ende des Stückes das Parlament Maria zur Prinzessin von Wales erheben will, lehnt sie es ab, die Enteignungen der Katholischen Kirche anzuerkennen: "Y pues vuestra majestad sabe la verdad, no quiera que por razones de Estado la ley de Dios se perviertas" ${ }^{\text {"t. }}$. So zeigt sich dem Publikum la ley de Dios der razón de Estado überlegen. Die Ordnung ist insofern wiederhergestellt, als die razón de Estado mit ihren unterschiedlichen Erscheinungsformen widerlegt worden ist.

Zusammenfassend lässt sich festhalten: Das mittelalterliche Modell ist von einer lex divina dominiert, aus der lex naturalis und lex positiva möglichst deduktiv abzuleiten sind. Sein weltliches Korrelat findet das Modell im Papst als oberstem Richter und Herrscher. Die Vorstellung eines nichtempirischen Gegenübers zu den positiven Gesetzen hatte schon Platon durch den Gegensatz von physis und thesis artikuliert und Aristoteles durch den Begriff der mesotes, der rechten Mitte, mit der Moral verbunden. Zudem hatte Aristoteles

ol Platon. Gorgicas, $483 \mathrm{c}-48+\mathrm{b}$.

6. Pedro Calderón de la Barca, "La cisma de Inglaterra», in: Obras completas (Anm. 42), S. $491-521$, hier S. 509.

o. Ehd., S. 512 .

of thd.. S. 520 . 
wie Thomas die Welt als vernünftige Ordnung verstanden, in der der Weltregent wie ein Künstler mit seiner ratio Ordnung als Zielursache vorgibt. Der Thomist Suárez entwickelt daraus eine Art Emanationsverhältnis, in dem aus dem göttlichen das natürliche und aus dem natürlichen das menschliche Gesetz hervorgeht, so dass zum Beispiel ein weiser Fürst in seiner Weisheit immer auch die lex divina einbezieht.

In der Theorie erwies sich das System als stimmig, in der Praxis jedoch zeigten sich Brüche. Wenn es dem Legislator nicht möglich war, alle möglichen Einzelfälle zu übersehen, wenn er Entwicklungen und Neuerungen nicht bemerkt, wenn er überfordert oder tyrannisch ist oder wenn das Volk zur Räuberbande wird, dann funktionieren die Ableitungen nicht mehr. Auch erwiesen sich Universitätsstatuten und Getreidepreise als von göttlichem und natürlichem Recht unberührte Gestaltungsfreiräume.

Allerdings sind dies nur praktische Aspekte, die die Gültigkeit der Theorie nicht tangieren. So wie Antigone die falschen Gesetze Kreons durch göttliche widerlegt sieht, sehen die Morisken positives durch göttliches Gesetz entkräftet. Und auch die Ermordung des Tyrannen Aurelian war durch höheres Gesetz legitimiert. Dadurch, dass die Konflikte am Ende jeweils zugunsten des höheren Gesetzes behoben sind, bestätigt sich in allen drei Beispielen die Dominanz der nichtempirischen Gesetzesebenen. Opposition gegen positives Recht legitimiert sich dabei durch göttliches Recht oder Naturrecht und kann sich auf Vitoria berufen, der den Volksaufstand mit dem Hinweis auf Petrus' Haltung gegenüber den Saduzäern und Pharisäern rechtfertigt, oder auf Thomas' Pauluszitat, nach dem Gott mehr zu gehorchen ist als den Menschen.

Das mittelalterliche Modell funktioniert also deduktiv durch Ableiten des positiven Gesetzes vom allgemeineren natürlichen oder göttlichen Gesetz. Gegen die Induktion vom positiv gegebenen Konkreten auf das Allgemeine führt Thomas an, dass der menschliche Wille dann nicht ungerecht sein könnte, wenn positives Recht schon deshalb gerecht wäre, weil es aus dem Willen der Menschen hervorgehe. Auch für die Stoiker ergab sich die Gesetzeshaftigkeit nicht aus den Willensbekundungen des Volkes. Eben die Deduktion aber muss bei Machiavelli wegfallen, da er den gesamten nichtempirischen Teil der Rechtstheorie streicht. Es bleibt das positive Gesetz. War zuvor die Finalursache der polis oder der res publica das bonum commune, wobei das bonum teleologisch als Zweck ${ }^{65}$ auch eine ethische Dimension hatte, wird das bonum commune nunmehr als Stärke verstanden, der Staatsräson zugeordnet und zum reinen Machtfaktor. Die Kategorie des bonum commune erweist sich als ausgehöhlt und bietet, wie auch die der consuetudo, nunmehr einen neuen Ausgangspunkt für induktives Denken.

65 Vgl. zur Bedeutung des Zwecks für die gesellschaftliche und rechtliche Ordnung bei Suárez Julius Seiler, Der Zweck in der Philosophie des Franz Suárez, Innsbruck: Rauch, 1936, S. 97-101. 
Das ebenso Hervorhebenswerte wie Problematische bei Machiavelli ist nun, dass durch Verlust des göttlichen und des natürlichen Rechts Teleologie und Deduktion gleichermaßen wegfallen und sich die Induktion auf die Optimierung der Mittel zur Machtvergrößerung beschränkt. Zu welchen negativen Entwicklungen eine individualisierte Staatsräson führt, wo sich der jeweils Stärkere nicht nur über göttliche und natürliche, sondern auch über positive Gesetze hinwegsetzt, hat Calderón in La cisma de Inglaterra gezeigt. Mit diesem Stück wird dem Machiavellismus und den Positionen des vorsokratischen Skeptikers Karneades und des Sophisten Kallikles ebenso widersprochen wie auch in neuerer Zeit durch Kant und im deutschen Grundgesetz, wenngleich in letzteren beiden Fällen die positive Gesetzgebung im a priori bzw. im Naturrecht verwurzelt ist ${ }^{66}$.

66 Vgl. Immanuel Kant, Metaphysische Anfangsgründe der Rechtslehre, hrsg. v. Bernd Ludwig, Hamburg: Meiner, 1986 (Philosophische Bibliothek, 360): «Das Naturrecht im Zustande einer bürgerlichen Verfassung (d. i. dasjenige, was für die letztere aus Prinzipien a priori abgeleitet werden kann) kann durch die statuarischen Gesetze der letzteren nicht Abbruch leiden» (S. 64). Naturrecht ist «das nicht-statuarische, mithin lediglich das a priori durch jedes Menschen Vernunft erkennbare Recht» (S. 109). Allgemein untergliedert er: «in das Naturrecht, das auf lauter Prinzipien a priori beruht, und das positive (statuarische) Recht, was aus dem Willen eines Gesetzgebers hervorgeht» (S. 46). - In der frühen Kommentierung des deutschen Grundgesetzes wurde angesichts des Nationalsozialismus und der Nürnberger Kriegsverbrecher-Prozesse der Artikel 1 («Die Würde des Menschen ist unantastbar») als naturrechtlich begründet angesehen. Das Naturrecht wird im Artikel 6 (2) explizit erwähnt: «Pflege und Erziehung der Kinder sind das natürliche Recht der Eltern [...]». Vgl. Gustav Radbruch, "Gesetzliches Unrecht und übergesetzliches Recht», in: Süddeutsche Juristenzeitung 1, 5 (1946), S. 105-108; zur neueren Kommentierung des Artikels 1 (1) durch Matthias Herdegen vgl. Ernst-Wolfgang Böckenförde, «Die Würde des Menschen war unantastban, in: Frankfurter Allgemeine Zeitung, 3. 9. 2003. 